\title{
miR-143 and miR-145 inhibit stem cell characteristics of PC-3 prostate cancer cells
}

\author{
SHUAI HUANG ${ }^{*}$, WEI GUO*, YUBO TANG, DONG REN, XUENONG ZOU and XINSHENG PENG \\ Department of Orthopaedic Surgery/Orthopaedic Research Institute, The First Affiliated Hospital \\ of Sun Yat-sen University, 510080 Guangzhou, Guangdong Province, P.R. China
}

Received July 3, 2012; Accepted August 21, 2012

DOI: $10.3892 /$ or.2012.2015

\begin{abstract}
Emerging evidence demonstrates that cancer stem cells (CSCs) are the critical drivers of tumor progression and metastasis. The microRNAs (miRNAs) may play a crucial role in repressing/promoting metastasis of cancer by regulating CSCs. A previous study showed that miR-143 and miR-145 play an important role in regulating bone metastasis of prostate cancer (PCa), but the exact mechanism of regulation of bone metastasis of PCa is not fully understood. In this study, we found that overexpression of miR-143 and miR-145 inhibited the cell viability and colony formation of PC-3 cells from PCa bone metastasis. Furthermore, miR-143 and miR-145 suppressed tumor sphere formation and expression of CSC markers and 'stemness' factors including CD133, CD44, Oct4, c-Myc and Klf4 in PC-3 cells. The study further found that miR-143 and miR-145 inhibit bone invasion and tumorigenicity of PC-3 cells in vivo. Collectively, these findings demonstrate that miR-143 and miR-145 inhibit CSC properties of PC-3 cells and suggest that miR-143 and miR-145 may play a significant role in the bone metastasis progression of PCa by regulating CSC characteristics.
\end{abstract}

\section{Introduction}

Prostate cancer (PCa) is the most frequently diagnosed cancer in men and the second leading cause of cancer death among men in the United States (1). The most common site of PCa metastasis is the bone, with bone metastases identified at autopsy in up to

Correspondence to: Dr Xinsheng Peng, Department of Orthopaedic Surgery, The First Affiliated Hospital of Sun Yat-sen University, No. 58 Zhongshan 2rd Road, 510080 Guangzhou, Guangdong Province, P.R. China

E-mail: pengxs66@yahoo.com

*Contributed equally

Abbreviations: PCa, prostate cancer; miRNA, microRNAs; CSCs, cancer stem cells; EMT, epithelial to mesenchymal transition; ESCs, embryonic stem cells

Key words: prostate cancer cells, microRNAs, cancer stem cell, stemness, bone metastasis
$90 \%$ of patients dying from PCa (2-4). However, the cellular and molecular mechanism underlying bone metastasis is relatively poorly understood, and more effective therapeutic strategies are clearly required in order to oppose PCa bone metastasis.

MicroRNAs (miRNAs) are a diverse family of small RNA molecules that function as a crucial post-transcriptional regulatory mechanism in various cellular functions $(5,6)$ and play a crucial role in tumor metastasis by regulating migration, invasion and epithelial to mesenchymal transition (EMT) (7-10). In PCa, a series of miRNAs were identified to regulate metastasis including miR-21 (11), miR-221 (12), miRNA-200 (13), miR-34a (14) and let-7 (15). We have previously identified that miR-143 and -145 repressed the ability of migration and invasion of PC-3 cells from PCa bone metastasis, and tumor development and bone invasion in vivo, and were negatively correlated to bone metastasis (16). Although we have further found that miR-143 and miR-145 may repress bone metastasis of PCa by regulating EMT (16), which is considered to be a crucial event in the metastatic process (17), the exact mechanisms of miR-143 and miR-145 regulating bone metastasis of $\mathrm{PCa}$ is not fully understood.

In recent years, accumulating evidence has provided support that a number of major cancers may be initiated by a small subset of cancer cells with stem cell properties, referred as cancer stem cells (CSCs), which display unlimited proliferation potential, ability to self-renew, and capacity to generate a progeny of differentiated cells that constitute the major tumor population (18-20). Emerging evidence demonstrates that CSCs might be the critical drivers of tumor progression and metastasis $(21,22)$. Furthermore, miRNAs also played a pivotal role in regulating the characteristics of CSCs by negatively regulating the expression of certain key genes in stem cells such as CD44, Oct4, Sox 2, c-Myc, and Klf4 (23). Therefore, miRNAs may play a crucial role in repressing/promoting metastasis of cancer by regulating CSCs. In $\mathrm{PCa}$, the recent studies have showed that let-7 (15), miRNA-200 (13) and miR-34a (14) may be important in the progression and metastasis of cancer by regulating CSCs. Because miR-145 regulated Oct4, Sox 2 and Klf4, and repressed pluripotency in human embryonic stem cells (ESCs) (24), at the same time, CSCs may share a degree of similarity with ESCs (25), miR-145 might regulate the stemness factors in PCa cells. Moreover, miR-143 and miR-145 may repress bone metastasis of PCa by regulating EMT (16), 
which is mechanistically linked with stem cell signatures in PCa (13). Thus, the above findings make us hypothesize that miR-143 and miR-145 might regulate stem cell characteristics of PCa cells.

In this study, to test the hypothesis, we used in vitro assays and in vivo xenograft models to examine the effects of miR-143 and miR-145 on stem cell characteristics of PCa bone metastasis PC-3 cells. The results demonstrated that both miR-143 and miR-145 inhibited CSC characteristics of PC-3 cells and suggest that they might be involved in the bone metastasis progression of $\mathrm{PCa}$ by regulating $\mathrm{CSC}$ characteristics.

\section{Materials and methods}

Cell culture and generation of stably transfected cell lines. The bone metastatic PCa cell line PC-3 was purchased from American Type Culture Collection (ATCC) and maintained in F-12 culture medium (Hyclone) supplemented with $10 \%$ fetal bovine serum (Hyclone). Stably-transfected cells were maintained in media with the presence of puromycin (SigmaAldrich). Cells were grown at a humidified atmosphere of 5\% $\mathrm{CO}_{2}$ at $37^{\circ} \mathrm{C}$. The sequence of pri-miR-143 and pri-miR-145 were cloned into pMSCV-puromycin plasmid with restriction enzyme $B g l \mathrm{II}$ and $E c o$ RI (New England Biolabs). 293FT cells were then transfected with the aforementioned constructed plasmids combined with PIK vector or blank pMSCV-vector as control, using the calcium phosphate method as described previously (26). After incubation at $37^{\circ} \mathrm{C}$ for $6 \mathrm{~h}$ after transfection, the media were changed and the cells were incubated overnight. To produce new virus, the media were collected thrice a day until 293FT cells reach to total confluence. Viruses were used to infect PC-3 cells. Twenty-four hours after addition of viruses, infected cells were selected by adding puromycin to growth medium. Stable cell lines were verified by qRT-PCR. Both pMSCV and PIK plasmids were generous gifts of Professor L.B. Song, Sun Yat-Sen University Cancer Center, Guangzhou, China.

Cell viability assay. Cell viability was determined by 2-(2-methoxy-4-nitrophenyl)-3-(4-nitrophenyl)-5-(2,4-disulfophen yl)-2H-tetrazolium, monosodium salt (WST-8) assay kit (CCK-8, Dojindo, Japan). Briefly, PC-3 cells were plated at a density of $5 \times 10^{3}$ cells/well in 96 -well plates and allowed to attach for $36 \mathrm{~h}, \mathrm{CCK}-8$ was used according to the manufacturer's instructions. WST- 8 was added into each well for 4 hours before the measurement. The absorbance at $450 \mathrm{~nm}$ was measured using a microplate reader.

Colony formation assay. Colony formation assay was performed as previously described (27). PC-3 cells were plated at 300 cells as single cells onto a $65-\mathrm{mm}$ Petri dish for 14 days, and colonies were stained with crystal violet. Plating efficiency $=$ number of colonies ( $\geq 50$ cells per colony) per input cells $x 100 \%$. To determine different colony morphologies, the different colony morphologies were scored under a light microscope.

Self-renewing spheroid formation assay. Spheroid formation assay was performed in PC-3/miR-143, PC-3/miR-145 and PC-3/ vector. Cells were plated at 400 cells/well onto 6 -well polyHEMA (Sigma)-coated plates and were grown in F12 medium
(Hycolone) for 14 days supplemented with B27 (Invitrogen), $20 \mathrm{ng} / \mathrm{ml}$ EGF (Sigma), and $20 \mathrm{ng} / \mathrm{ml}$ basic FGF (Invitrogen). After 14 days, the number of prostaspheres (tight, spherical, nonadherent masses $>100 \mu \mathrm{m}$ in diameter) were counted, and image of the prostaspheres were captured under inverse microscope. Sphere formation efficiency $=$ colonies/input cells $\mathrm{x} 100 \%$.

Western blot assay. Western blotting was performed as previously described (16). Briefly, cells were lysed with sample buffer [62.5 mmol/1 Tris-HCl (pH 6.8), 2\% SDS, 10\% glycerol, and $5 \% \quad 2-\beta$-mercaptoethanol]. Proteins were resolved in SDS-polyacrylamide gel by electrophoresis and then transferred onto Hybond-P PVDF membrane (Amersham Biosciences, Piscataway, NJ). Antibodies used were anti-Oct4, anti-Sox2, anti-c-Myc, and anti-Klf4 (Cell Signaling, Technology Inc., Beverly, MA), CD133 (Miltenyi Biotech, Auburn, CA), CD44 (Santa Cruz Biotechnology, Santa Cruz, CA). After washing with TBS-T, the membrane was incubated with anti-rabbit IgG secondary antibodies, and the signals were visualized using the ECL plus western blotting system (Amersham).

In vivo tumorigenicity assay. To determine whether miR-143 and miR-145 can inhibit tumor development, we manipulated miR-143 and miR-145 levels in PCa PC-3 cells and then implanted the cells into the NOD-SCID mice. Intra-tibial injection model was used. Twelve male severe combined immunodeficient (SCID) mice, 3-4 weeks old, were purchased from HFK Bio-Technology, Co., Ltd. (Beijing, China). Intratibial injection was performed as previously described (16). Mice were monitored weekly for tumor growth. On week 5, hind limbs were radiographed using a Faxitron X-ray machine (Faxitron X-ray Corp., USA) to detect the bone lesions. Then mice were sacrificed, and tibias were collected, decalcified and fixed in formalin for further histologic analysis. Bone lesions were evaluated and calculated as described as previously described $(16,28)$. The animals were sacrificed 5 weeks after receiving radiograph. All tumors were resected at autopsy and sectioned for histological analysis. The animal study was approved by the Institutional Ethical Board (IRB) in the First Affiliated Hospital of Sun Yat-sen University.

Statistical analysis. Experimental data were expressed as mean \pm standard deviation (SD). One-way ANOVA was performed for comparing more than two groups, and paired Student's t-test was performed for comparing X-ray scores. Statistical analyses were assessed using SPSS 17.0 (SPSS, Inc., Chicago, IL, USA). Statistical significance was accepted at $\mathrm{p}<0.05$.

\section{Results}

miR-143 and miR-145 inhibits cell viability. The miR-143 and miR-145 overexpressing cell lines (PC-3/miR-143,PC-3/miR-145) were established by retrovirus transfection (16). Blank plasmid transfected cells, PC-3/vector were used as control group. To test if miR-143 and miR-145 decrease cell viability of PC-3 cells, cell viability of PC-3/miR-143, PC-3/miR-145 and PC-3/vector was examined by CCK8 assay for $36 \mathrm{~h}$. The results showed that miR-143 and miR-145 significantly reduced cell viability (PC-3/ miR-143 compared with PC-3/vector, $\mathrm{p}<0.01$; PC-3/miR-145, compared with PC-3/vector, $\mathrm{p}<0.01$ ) (Fig. 1). 


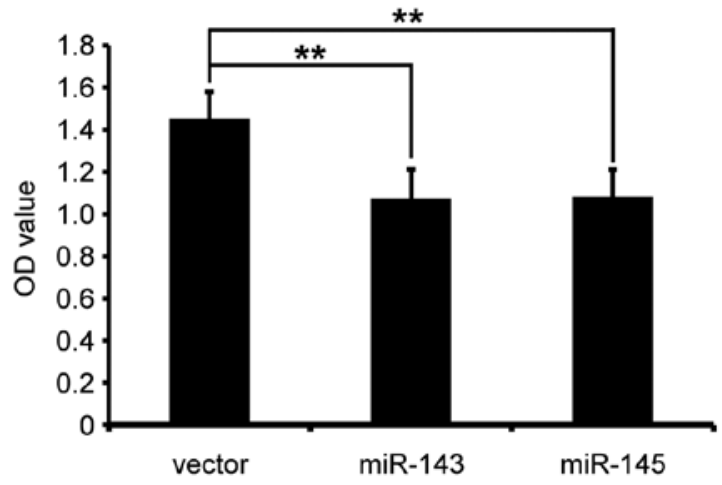

Figure 1. miR-143 and miR-145 inhibit cell viability. The OD value showed that PC-3/miR-143 and PC-3/miR-145 cell viability decreased significantly compared with PC-3/vector ( ${ }^{* *} \mathrm{p}<0.01$, respectively).

miR-143 and miR-145 inhibits colony formation in PC-3. To determine efficiency of miR-143 and miR-145 inhibiting colonyforming of PC-3 in vitro, colony-forming assay was performed in PC-3/miR-143, PC-3/miR-145 and PC-3/vector. The number of colonies (\% plating efficiency) were $33.25 \pm 4.92 \%$ in PC-3/ $\mathrm{miR}-143,31.75 \pm 5.56 \%$ in PC-3/miR-145, and $59.75 \pm 4.42 \%$ in $\mathrm{PC}-3 /$ vector, and significantly decreased in PC-3/miR-143 and PC-3/miR-145 compared with PC-3/vector $(\mathrm{p}<0.01$, respectively) (Fig. 2A and C). Colonies with different morphologies in vitro are classified as holoclones, meroclones, and paraclones (27). Holoclones are generally more round and tightly packed, and paraclones are irregular in composition and often contain more elongated or flattened cells, and meroclones are an intermediate phenotype. We only found meroclones and paraclones in PC-3 cells (Fig. 2B). The proportion of meroclones was $44.25 \pm 7.46 \%$ in PC- $3 / \mathrm{miR}-143,44.75 \pm 5.90 \%$ in PC-3/miR- 145 , and $64.75 \pm 5.50 \%$ in PC-3/vector the miR-143 and miR-145 significantly decreased the proportion of meroclones of PC-3 cells $(\mathrm{p}<0.05)$ (Fig. 2D).

miR-143 and miR-145 inhibit tumor spheroid formation. The ability to grow as non-adherent spheroids in the sphere medium has been widely used to assess the self-renewal capability of CSCs and is one of the characteristics of prostate CSCs $(20,29)$.

\section{A}

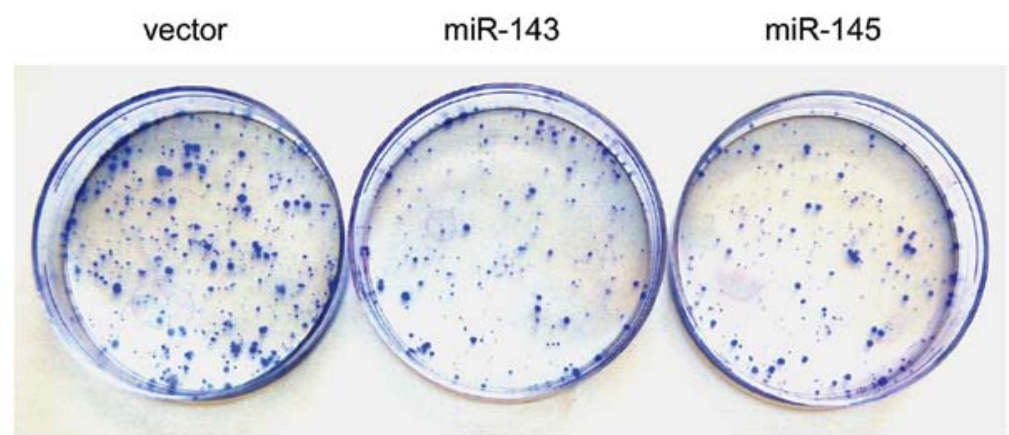

B
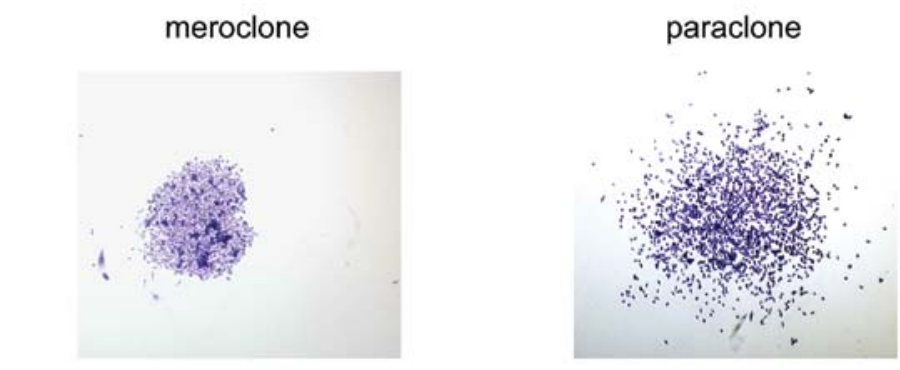

C
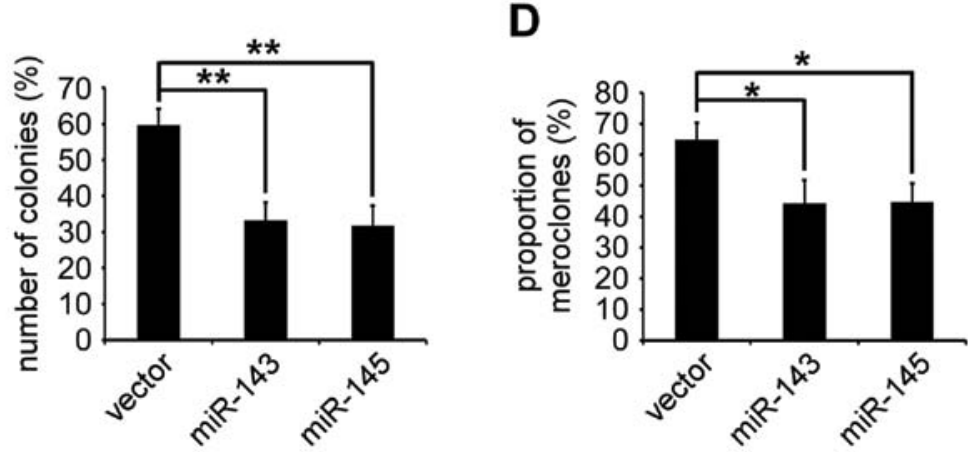

Figure 2. miR-143 and miR-145 inhibits colony-forming efficiency in PC-3 cells. (A) Colony-formation exists in PC-3/miR-143, PC-3/miR-145 and PC-3/vector cells. (B) A typical holoclone phenotype was not observed, and only meroclones and paraclones were detected. (C) PC-3/miR-143 and PC-3/miR-145 significantly repressed the number of colonies ( ${ }^{*} \mathrm{p}<0.05$, respectively). (D) PC-3/miR-143 and PC-3/miR-145 significantly suppressed the proportion of meroclones ${ }^{* *} \mathrm{p}<0.01$, respectively). 
A

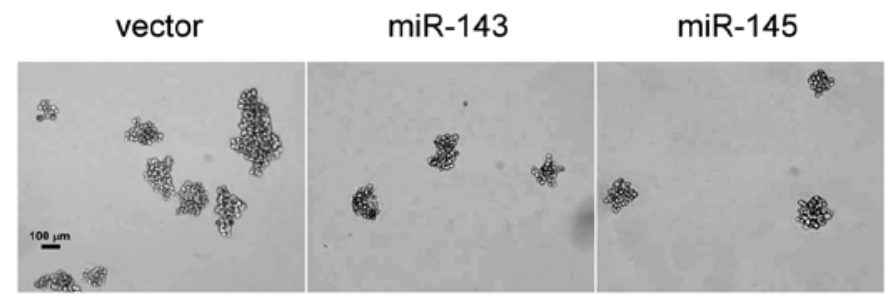

B

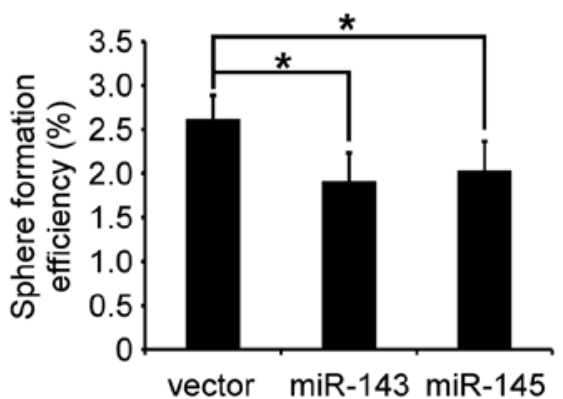

Figure 3. miR-143 and miR-145 suppress tumor sphere formation. Four hundred cells were seeded onto polyHEMA pre-coated plates for 14 days. (A) The tumor sphere formation existed in PC-3/miR-143, PC-3/miR-145 and PC-3/vector cells. (B) miRs-143 and miR-145 efficiently suppresses the spheroid formation ability of PC-3 cells ( ${ }^{*} \mathrm{p}<0.05$ respectively).

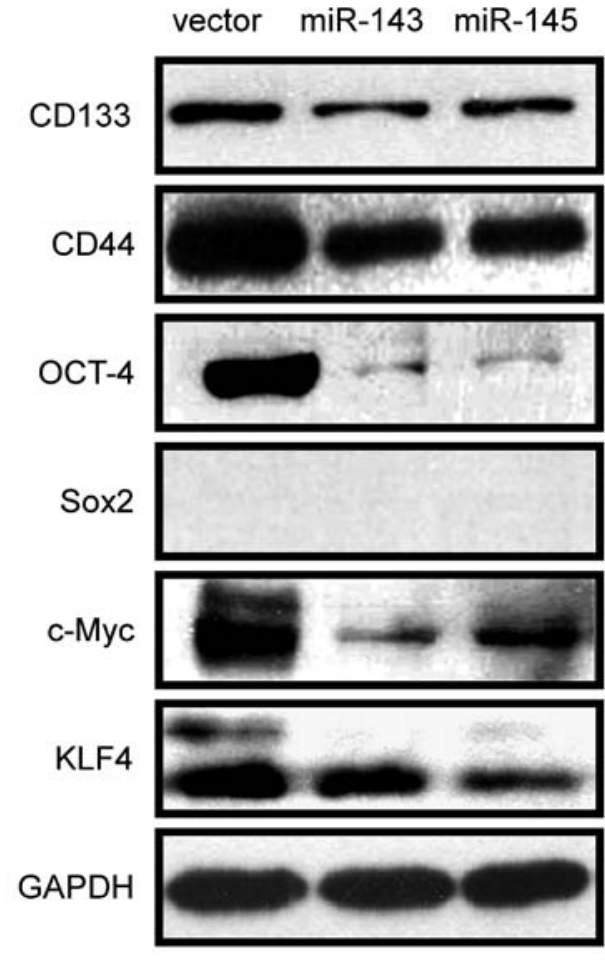

Figure 4. miR-143 and miR-145 inhibit CSC marker and stemness factor expression. CD133, CD44, OCT4, SOX2, C-MYC and KLF4 were detected by western blotting in PC-3/miR-143, PC-3/miR-145 and PC-3/vector. Downregulation of CD133, CD44, OCT4, C-MYC and KLF4CD133, KLF4 was observed in PC-3/miR-143, PC-3/miR-145 compared with PC-3/vector, but SOX2 was not detected in PC-3 cells.

To confirm that miR-143 and miR-145 can inhibit the selfrenewal capability of PC-3 cells, prostasphere formation of PC-3 cells was studied. As shown in Fig. 3, after culturing for 14 days under non-adherent conditions, there were prostaspheres in all the three kinds of cells. The spheroid formation efficiency was $2.618 \pm 0.27 \%$ in PC-3/vector, $1.915 \pm 0.32 \%$ in PC-3/miR-143 and $2.034 \pm 0.33 \%$ in $\mathrm{PC}-3 / \mathrm{miR}-145$, confirming the presence of the self-renewal cells in PC-3/miR-143, PC-3/miR-145 and PC-3/ vector. Further, both miR-143 and miR-145 suppressed significantly prostasphere formation ( $\mathrm{p}<0.05$, respectively). This result indicated that miR-143 and miR-145 repressed CSCs properties of PC-3 cells.
miR-143 and miR-145 inhibit CSC marker and stemness factor expression. Because CD133 and CD44 have been described as prostate CSC markers based on clinical investigations and in vitro studies of prostate cancer cell lines (14,30-32), we first investigated if miR-143 and miR-145 repressed the expression in PC-3 cells. The expression of CD133 and CD44 was examined by western blotting. As shown in Fig. 4, overexpression of miR-143 and miR-145 repressed the expression of CD133 and CD44. Furthermore, since transcription factors Oct-4, Sox-2, c-Myc and KLF4 are the key stemness factors and are required for maintaining self-renewal and pluripotency of stem cells $(24,33)$, we sought to determine whether miR-143 and miR-145 regulate the expression of these stemness factors. As shown in Fig. 4, overexpression of miR-143 and miR-145 downregulated the expression of Oct4, c-Myc and Klf4, but Sox 2 was not detected in PC-3/miR-143, PC-3/miR-145 and PC-3/vector. These results suggested that miR-143 and miR-145 might modulate CSCs properties in PC-3 cells by regulating CD133, CD44, Oct4, c-Myc and Klf4.

miR-143 and miR-145 inhibit tumorigenicity in vivo. To determine if miR-143 and miR-145 repressed tumorigenicity in vivo, male SCID mice were inoculated with PC-3/miR-143, PC-3/ miR-145 and PC-3/vector cells through the intra-tibial route. Five weeks after inoculation, as showed in Fig. 5A and B, skeletal lesions in the left tibias were obviously larger than those in the right tibias, which mean that PC-3/miR-143 and PC-3/ miR-145 had less skeletal invasion and tumorigenicity ability than PC-3/vector. H\&E-staining was performed as histological confirmation. The extent and areas of skeletal lesions were assessed by X-ray scores, and PC-3/miR-143 and PC-3/miR-145 showed significantly less ability in forming tumors and bone invasion compared with PC-3/vector ( $\mathrm{p}<0.05$, respectively). The results suggested that miR-143 and miR-145 could repress bone invasion and tumorigenicity.

\section{Discussion}

In this study, we found that miR-143 and miR-145 inhibited the cell viability, suppressed colony formation and repressed tumor sphere formation of PC-3 cells from PCa bone metastasis. Furthermore, miR-143 and miR-145 repressed expression of CSC markers and stemness factors including CD133, CD44, Oct4, c-Myc and Klf4 in PC-3 cells. Both 
A
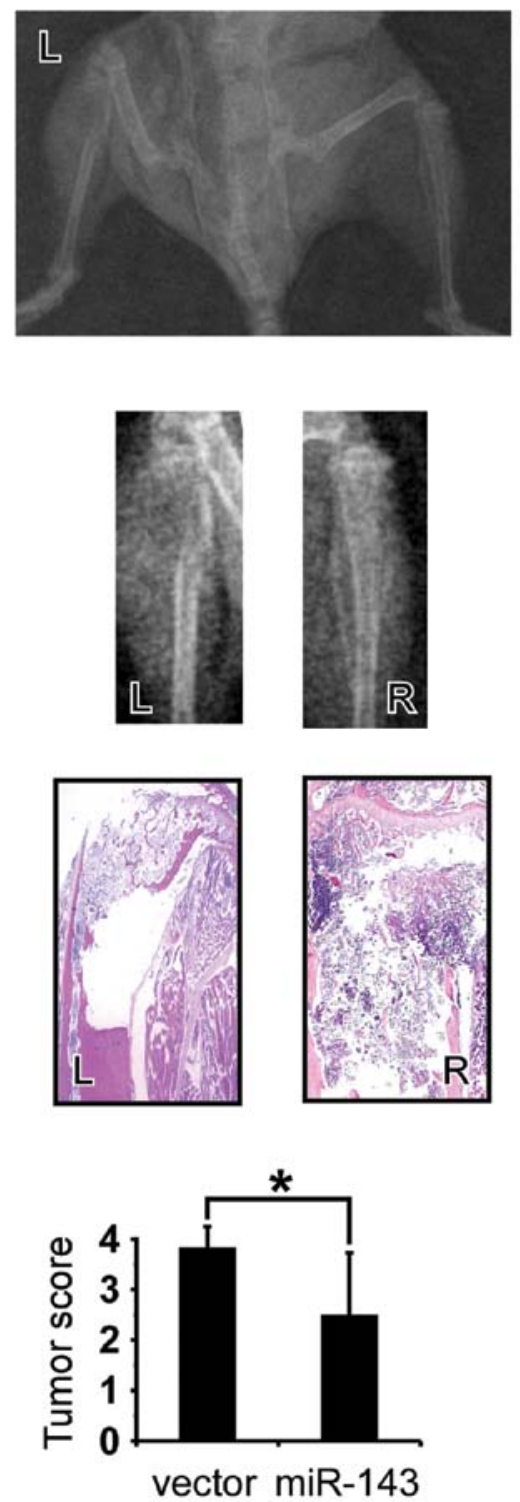

B
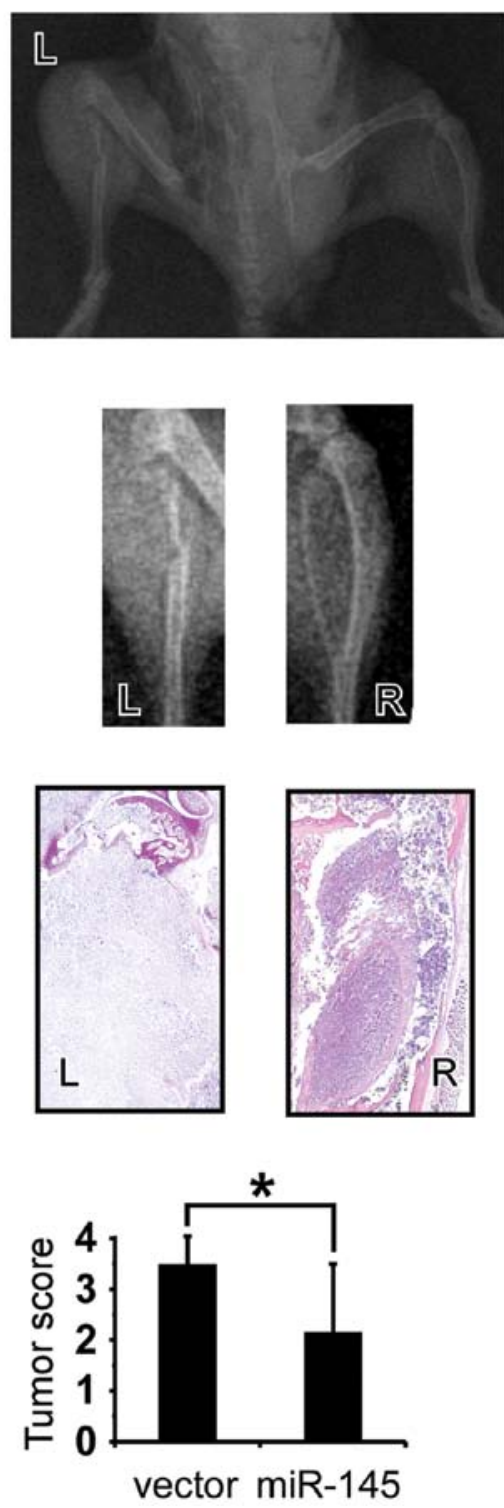

Figure 5. miR-143 and miR-145 inhibit tumorigenicity of PC-3 cells in vivo. Skeletal lesions in the left tibias were obviously larger than those in the right tibias (top panel), which means PC-3/miR-143 and PC-3/miR-145 had less skeletal invasion and tumorigenic ability than PC-3/vector (second panel). H\&E-stainning was performed as histological confirmation (third panel). The extents and areas of skeletal lesions were assessed by X-ray scores (bottom panel), and PC-3/ miR-143 and PC-3/miR-145 showed significantly less ability in forming tumors and bone invasion compared with PC-3/vector ( ${ }^{*} \mathrm{p}<0.05$, respectively).

inhibited bone invasion and tumorigenicity of PC-3 cells in NOD-SCID mice tibia. These findings demonstrate that miR-143 and miR-145 negatively regulate the CSCs properties of PC-3 cells from PCa bone metastasis. Importantly, CSCs may be the critical drivers of tumor progression and metastasis $(21,22)$. Thus, our results suggest that miR-143 and miR-145 might play a significant role in the bone metastasis progression of $\mathrm{PCa}$ by regulating CSC characteristics.

Emerging evidence suggests that miRNAs may function as the regulators of CSC characteristics in many studies (13-16,23,34). In PCa, let-7 inhibited self-renewal and clonogenic capacity of cancer cells by directly targeting EZH2 (15). The miR-34a was established as an important negative regulator of $\mathrm{CD}_{4} 4^{+} \mathrm{PCa}$ cells (putative CSCs) and is involved in PCa development and metastasis (14). The role of miR-34a in controlling CSC characteristics appears to be important by directly repressing CD44 expression in $\mathrm{PCa}$ (14). In this study, we found miR-143 and miR-145 also can repressed the expression of CD44, which is speculated as one of miR-143 and miR-145 putative targets (miRWalk) and is the most common of CSC markers (32). Previously, CD $44^{+} \mathrm{PCa}$ cells were shown to have the stem-like properties of increased tumorigenic, clonogenic, and metastatic potential (30). Although CD44 does not seem to belong to the stemness genes, such as Oct4 and $\mathrm{Klf} 4$, that are central for maintaining stem cell characteristics, CD44 can contribute to the activation of stem cell regulatory genes and can be a target of these genes (32). More importantly, a recent study has demonstrated that the transcriptional reprogramming led by nuclear $\mathrm{CD} 44$ has an active role in transforming cancer cells to a CSC-like phenotype (17). Therefore, our finding suggested that miR-143 and miR-145 may possess a similar function with miR-34a in controlling 
CSC characteristics of $\mathrm{PCa}$, and regulate metastasis of $\mathrm{PCa}$ by targeting CD44.

Previously, it was found that miR-145 directly targets the 3'UTRs of the stemness factors Oct4, Sox2, and Klf4 in ESCs (24) and the stemness factor c-Myc also is a direct target for miR-145 (35). Yang et al (36) and Chiou et al (37) demonstrated that miR145 repressed CSCs characteristics by targeting Oct4 and Sox 2 in glioblastoma-CD133 ${ }^{+}$and lung adenocarcinomaassociated CSCs. Our results showed that miR-145 repressed the expression of Oct4, c-Myc and Klf4 of PC-3 cells. Thus, miR-145 may regulate CSC characteristics of PC-3 cells, at least in part, by directly targeting Oct4, c-Myc, and Klf4. In this study, the results also showed that miR-143 play a similar role to miR-145 regulating CSC characteristics of PC-3 cells and repressed the expression of Oct4, c-Myc, and Klf4. However, how miR-143 regulate the stemness factors and the exact mechanism of miR-143 regulation of CSC characteristics need to be further explored. We did not detect the expression of Sox 2 in PC-3 cells by western blot analysis. The transcripts for Sox 2 were not detected in the PC-3 cells by reverse transcription (RT)-PCR (38). Thus, Sox 2 may not be the factor regulated by miR-143 and miR-145 in PC-3 cells.

CSCs and EMT-type cells have been proposed to play critical roles in cancer metastasis as demonstrated in several human malignancies (39). Recent evidence has demonstrated that the EMT can generate cancer cells with properties of stem cells $(13,40-42)$. This important finding implies a direct link between EMT and cancer stem cells. Thus, the discovery of molecular knowledge related to CSC characteristics and EMT in PCa is important. Previously it was found that miR-200 and let-7 played a critical role in linking EMT phenotype with stem cell signatures by regulating the expression of Lin28B and Notch1 (13). In this study, we found that miR-143 and miR-145 regulated CSC characteristics of PCa. Importantly, our previous study found that overexpression of miR-143 and miR-145 repressed EMT of PC-3 cells of PCa (16). Therefore, miR-143 and miR-145 might be the new links between the characteristics of cancer stem-like cells and EMT in PC-3 cells.

In our study, morphologically typical holoclones were not detected in PC-3 cells. The results of Pfeiffer and Schalken (27) are in agreement with ours. However, other studies showed that the number of holoclones formed by PC-3 cells was composed of approximately $10 \%$ of all clones $(43,44)$. The main reason for this phenomenon is that higher plating density was adopted in our and Pfeiffer and Schalken studies compared with previous report in which PC3 cells were plated under diluted conditions ( $\sim 1$ cell per well) $(44,45)$. Moreover, it was demonstrated earlier that PC-3 cells had an intrinsic impaired cell-cell adhesion because of the E-cadherin and associated $\alpha$-catenin were frequently reduced or absent in this cells, thus, they were not able to form tightly packed colonies (45). Additionally, continuous and rapid change of different colony types made the colonies difficult to distinguish from each other $(27,45)$. The definition of the three colony morphologies differs somewhat from each other, and there are no strict borderlines between the colony types, which makes the grading fairly subjective (27).

In conclusion, we have demonstrated, for the first time, that miR-143 and miR-145 inhibit CSC properties of PC-3 cells from PCa bone metastasis. Our findings suggest that miR-143 and miR-145 might inhibit the bone metastasis progression of
PCa by repressing CSC characteristics, and might hold significant promise as a new class of molecular therapy for human PCa bone metastasis, potentially by modulating cancer stem cells.

\section{Acknowledgements}

We thank Dr Wenjian Wang, Dr Longjuan Zhang and Dr Wen Li from the Surgical Laboratory at The First Affiliated Hospital of Sun Yat-sen University for their excellent technical help. We also thank NSFC-Guangdong Joint funding, China (No. u0732001); Science and Technology planning project of Guangdong Province, China (No. 2008B030301037), Science and Technology Planning Project of Guangzhou, China (11C22060772) and Science and Technology Planning Project of Zhuhai, China (2009) for supporting this study.

\section{References}

1. Landis SH, Murray T, Bolden S and Wingo PA: Cancer statistics, 1999. CA Cancer J Clin 49: 8-31, 1999.

2. Bubendorf L, Schopfer A, Wagner U, et al: Metastatic patterns of prostate cancer: an autopsy study of 1,589 patients. Hum Pathol 31: 578-583, 2000.

3. Shah RB, Mehra R, Chinnaiyan AM, et al: Androgen-independent prostate cancer is a heterogeneous group of diseases: lessons from a rapid autopsy program. Cancer Res 64: 9209-9216, 2004.

4. Rana A, Chisholm GD, Khan M, Sekharjit SS, Merrick MV and Elton RA: Patterns of bone metastasis and their prognostic significance in patients with carcinoma of the prostate. Br J Urol 72: 933-936, 1993

5. Inui M, Martello G and Piccolo S: MicroRNA control of signal transduction. Nat Rev Mol Cell Biol 11: 252-263, 2010.

6. Schickel R, Boyerinas B, Park SM and Peter ME: MicroRNAs: key players in the immune system, differentiation, tumorigenesis and cell death. Oncogene 27: 5959-5974, 2008.

7. Baranwal S and Alahari SK: miRNA control of tumor cell invasion and metastasis. Int J Cancer 126: 1283-1290, 2010.

8. Garzon R, Calin GA and Croce CM: MicroRNAs in cancer. Annu Rev Med 60: 167-179, 2009

9. Khew-Goodall Y and Goodall GJ: Myc-modulated miR-9 makes more metastases. Nat Cell Biol 12: 209-211, 2010.

10. Nicoloso MS, Spizzo R, Shimizu M, Rossi S and Calin GA: MicroRNAs - the micro steering wheel of tumour metastases. Nat Rev Cancer 9: 293-302, 2009.

11. Li T, Li D, Sha J, Sun P and Huang Y: MicroRNA-21 directly targets MARCKS and promotes apoptosis resistance and invasion in prostate cancer cells. Biochem Biophys Res Commun 383: 280-285, 2009.

12. Spahn M, Kneitz S, Scholz CJ, et al: Expression of microRNA-221 is progressively reduced in aggressive prostate cancer and metastasis and predicts clinical recurrence. Int J Cancer 127: 394-403, 2010.

13. Kong D, Banerjee S, Ahmad A, et al: Epithelial to mesenchymal transition is mechanistically linked with stem cell signatures in prostate cancer cells. PLoS One 5: e12445, 2010.

14. Liu C, Kelnar K, Liu B, et al: The microRNA miR-34a inhibits prostate cancer stem cells and metastasis by directly repressing CD44. Nat Med 17: 211-215, 2011.

15. Kong D, Heath E, Chen W, et al: Loss of let-7 up-regulates EZH2 in prostate cancer consistent with the acquisition of cancer stem cell signatures that are attenuated by BR-DIM. PLoS One 7: e33729. 2012

16. Peng X, Guo W, Liu T, et al: Identification of miRs-143 and -145 that is associated with bone metastasis of prostate cancer and involved in the regulation of EMT. PLoS One 6: e20341, 2011.

17. Su YJ, Lai HM, Chang YW, Chen GY and Lee JL: Direct reprogramming of stem cell properties in colon cancer cells by CD44. EMBO J 30: 3186-3199, 2011.

18. Clarke MF, Dick JE, Dirks PB, et al: Cancer stem cells perspectives on current status and future directions: AACR Workshop on cancer stem cells. Cancer Res 66: 9339-9344, 2006. 
19. Sun S and Wang Z: ALDH high adenoid cystic carcinoma cells display cancer stem cell properties and are responsible for mediating metastasis. Biochem Biophys Res Commun 396: 843-848, 2010.

20. Visvader JE and Lindeman GJ: Cancer stem cells in solid tumours: accumulating evidence and unresolved questions. Nat Rev Cancer 8: 755-768, 2008

21. Chaffer CL and Weinberg RA: A perspective on cancer cell metastasis. Science 331: 1559-1564, 2011.

22. Monteiro $\mathbf{J}$ and Fodde R: Cancer stemness and metastasis: therapeutic consequences and perspectives. Eur J Cancer 46 : $1198-1203,2010$

23. Hatfield S and Ruohola-Baker H: microRNA and stem cell function. Cell Tissue Res 331: 57-66, 2008.

24. Xu N, Papagiannakopoulos T, Pan G, Thomson JA and Kosik KS MicroRNA-145 regulates OCT-4, SOX2, and KLF4 and represses pluripotency in human embryonic stem cells. Cell 137: 647-658, 2009.

25. Wong DJ, Segal E and Chang HY: Stemness, cancer and cancer stem cells. Cell Cycle 7: 3622-3624, 2008.

26. Zhang S, Balch C, Chan MW, et al: Identification and characterization of ovarian cancer-initiating cells from primary human tumors. Cancer Res 68: 4311-4320, 2008.

27. Pfeiffer MJ and Schalken JA: Stem cell characteristics in prostate cancer cell lines. Eur Urol 57: 246-254, 2010.

28. Yang M, Burton DW, Geller J, et al: The bisphosphonate olpadronate inhibits skeletal prostate cancer progression in a green fluorescent protein nude mouse model. Clin Cancer Res 12 : 2602-2606, 2006.

29. Bisson I and Prowse DM: WNT signaling regulates self-renewal and differentiation of prostate cancer cells with stem cell characteristics. Cell Res 19: 683-697, 2009.

30. Patrawala L, Calhoun T, Schneider-Broussard R, et al: Highly purified $\mathrm{CD} 44^{+}$prostate cancer cells from xenograft human tumors are enriched in tumorigenic and metastatic progenitor cells. Oncogene 25: 1696-1708, 2006.

31. Trerotola M, Rathore S, Goel HL, et al: CD133, Trop-2 and alpha2beta1 integrin surface receptors as markers of putative human prostate cancer stem cells. Am J Transl Res 2: 135-144, 2010.

32. Zöller M: CD44: can a cancer-initiating cell profit from an abundantly expressed molecule? Nat Rev Cancer 11: 254-267, 2011.

33. Schoenhals M, Kassambara A, De Vos J, Hose D, Moreaux J and Klein B: Embryonic stem cell markers expression in cancers. Biochem Biophys Res Commun 383: 157-162, 2009.

34. Liu C and Tang DG: MicroRNA regulation of cancer stem cells. Cancer Res 71: 5950-5954, 2011.
35. Sachdeva M, Zhu S, Wu F, et al: p53 represses c-Myc through induction of the tumor suppressor miR-145. Proc Natl Aca Sci USA 106: 3207-3212, 2009.

36. Yang YP, Chien Y, Chiou GY, Cherng JY, Wang ML, Lo WL, et al: Inhibition of cancer stem cell-like properties and reduced chemoradioresistance of glioblastoma using microRNA145 with cationic polyurethane-short branch PEI. Biomaterials 33: 14621476, 2012.

37. Chiou GY, Cherng JY, Hsu HS, et al: Cationic polyurethanesshort branch PEI-mediated delivery of Mir145 inhibited epithelial-mesenchymal transdifferentiation and cancer stem-like properties and in lung adenocarcinoma. J Control Release 159: 240-250, 2012

38. Lee MY, Lu A and Gudas LJ: Transcriptional regulation of Rex1 (zfp42) in normal prostate epithelial cells and prostate cancer cells. J Cell Physiol 224: 17-27, 2010.

39. Hayashida T, Jinno H, Kitagawa Y and Kitajima M: Cooperation of cancer stem cell properties and epithelial-mesenchymal transition in the establishment of breast cancer metastasis. J Oncol 2011: 591427, 2011

40. Mani SA, Guo W, Liao MJ, et al: The epithelial-mesenchymal transition generates cells with properties of stem cells. Cell 133: 704-715, 2008

41. Morel AP, Lièvre M, Thomas C, Hinkal G, Ansieau S and Puisieux A: Generation of breast cancer stem cells through epithelial-mesenchymal transition. PLoS One 3: e2888, 2008.

42. Pirozzi G, Tirino V, Camerlingo R, et al: Epithelial to mesenchymal transition by TGF $\beta-1$ induction increases stemness characteristics in primary non small cell lung cancer cell line. PLoS One 6: e21548, 2011.

43. Li H, Chen X, Calhoun-Davis T, Claypool K and Tang DG: PC3 human prostate carcinoma cell holoclones contain self-renewing tumor-initiating cells. Cancer Res 68: 1820-1825, 2008.

44. Zhang $\mathrm{K}$ and Waxman DJ: PC3 prostate tumor-initiating cells with molecular profile FAM65B $\mathrm{B}^{\text {high }} / \mathrm{MFI} 2^{\text {low }} / \mathrm{LEF} 1^{\text {low }}$ increase tumor angiogenesis. Mol Cancer 9: 319, 2010.

45. Morton RA, Ewing CM, Nagafuchi A, Tsukita S and Isaacs WB: Reduction of E-cadherin levels and deletion of the alpha-catenin gene in human prostate cancer cells. Cancer Res 53: 3585-3590, 1993. 